I NRA Prod. Anim., 2001, 14 (5), 365-370

\section{LESSIRE}

INRA, Stati on de Recherches Avi coles, 37380 Nouzi lly

Courrie : lessi re@tours.inra.fr

\title{
Matières grasses alimentaires et composition lipidique des volailles
}

L'état d'engraissement des carcasses de volailles varie selon l'espèce aviaire, le sexe et l'âge de l'oiseau, mais aussi selon les caractéristiques nutritionnelles de l'aliment ingéré. Les lipides ajoutés à l'aliment n'ont qu'une influence modérée sur la quantité de lipides déposés, mais ils peuvent modifier profondément la nature des acides gras de la carcasse.

La qualité des produits est une des préoccupations majeures des différents maillons de la filière avicole et du consommateur. Parmi les facteurs susceptibles d'altérer ou d'améliorer cette qualité on évoque souvent l'alimentation, même si d'autres paramètres ont une influence bien supérieure : le génotype, l'âge (Alleman et al 1999, Berri et Jehl 2001). De nombreuses revues et articles ont été consacrés à l'influence de l'alimentation sur la composition corporelle des espèces aviaires et en particulier au rôle des lipides alimentaires (Fisher 1984, Lessire 1995, Gandemer et al 1999, Viau et al 1999). L'introduction de lipides dans l'aliment des oiseaux répond à toute une série d'objectifs tant nutritionnels que technologiques. Leur addition permet en effet d'accroître la densité énergétique de la ration tout en permettant I'utilisation d'ingrédients à faible teneur en énergie, d'apporter aux oiseaux les acides gras indispensables et, lors de la fabrication

\section{Résumé}

Les viandes de volailles sont appréciées du consommateur et recommandées par les diététiciens parce qu'elles sont pauvres en lipides et malgré tout bien pourvues en acides gras insaturés. Selon les espèces aviaires, l'âge et le sexe, cette faible teneur en lipides est très relative et l'état d'adiposité peut aussi varier en fonction de nombreux critères liés à l'aliment. Les lipides ajoutés à l'aliment ont pour but principal d'accroître sa concentration énergétique, améliorant ainsi les performances de production. Leur incidence sur l'état d'engraissement des carcasses est mineure lorsque les équilibres nutritionnels et, en particulier, le rapport protéines sur énergie sont maintenus constants. Mais la nature des lipides ajoutés modifie de façon profonde celle des lipides corporels. Ainsi, il est possible d'adapter les profils des acides gras corporels des volailles, et du poulet en particulier, aux exigences de qualité des abattoirs et du consommateur. Cet article illustre les variations que peut présenter le profil en acides gras des tissus des volailles en relation avec les lipides ingérés. des aliments, de limiter les poussières et I'usure des machines. Cependant, ces lipides sont souvent accusés de provoquer un engraissement excessif des animaux, ce qui déprécie la carcasse, en particulier lorsque les dépôts adipeux visibles sont importants (gras abdominal, sous cutané, ... ).

L'excès de dépôts adipeux génère une diminution des rendements lors de l'éviscération, de la découpe et de l'élaboration des produits. Enfin, d'un point de vue nutritionnel, la synthèse et le dépôt de $1 \mathrm{~g}$ de lipides corporels sont plus coûteux que la synthèse et le dépôt de $1 \mathrm{~g}$ de protéines musculaires.

Le consommateur apprécie peu des dépôts adipeux importants, mais les lipides corporels ont un effet positif sur la qualité organoleptique des produits.

L'objectif de cet article est de présenter les facteurs de variation de la composition lipidique des viandes de volaille. Nous rapporterons quelques chiffres relatifs à la proportion de lipides que renferment les carcasses de volailles et mentionnerons les principaux facteurs de variation. Ensuite nous présenterons l'incidence des lipides alimentaires sur la nature des lipides déposés.

\section{1 / Composition lipidique des volailles}

Parmi les différentes espèces aviaires, le canard à rôtir dit 'maigre' présente, à l'âge d'abattage, la teneur en lipides corporels la plus élevée (18\%). A l'inverse, le dindon peut être considéré comme maigre $(10 \%)$ et la 
teneur en lipides du poulet est proche de celle du canard (17,7\%) (Larbier et Leclercq 1992). Pour une même espèce et à âge identique, les femelles sont généralement plus grasses que les mâles. Chez le canard, la différence d'engraissement entre les deux sexes est minime alors qu'elle s'élève à $21 \%$ chez le poulet de 42 jours et atteint $121 \%$ chez le dindon âgé de 112 jours.

D'une façon générale,l'état d'engraissement augmente régulièrement avec l'âge, exception faite du dindonneau dont la carcasse reste très maigre jusqu'à 7 semaines, elle ne renferme alors que $5 \%$ de lipides, puis la teneur en lipides s'accroît très rapidement pour atteindre $15,7 \%$ chez la femelle, alors que le mâle reste relativement maigre $(7,1 \%)$.

La répartition des masses adipeuses varie également selon les espèces aviaires (Leclercq 1989). Ainsi la proportion de gras abdominal est similaire chez le canard et le poulet ( 3 à $4 \%$ du poids vif), alors que la carcasse du dindonneau ne renferme que 1 à $2 \%$ de gras abdominal (tableau 1 ). Ce dépôt lipidique est éliminé lors de l'éviscération et constitue une perte à l'abattage. II s'agit d'un dépôt tardif utilisé comme critère de sélection aussi bien pour des lignées expérimentales maigres ou grasses que pour des croisements commerciaux. En effet, la sélection intense sur la vitesse de croissance induit un accroissement général de l'adiposité (Leclercq 1989) et la prise en compte du critère 'gras abdominal' permet de maintenir l'engraissement dans des limites raisonnables.

Enfin, la quantité de lipides varie également selon les tissus. Les muscles pectoraux blancs, ou filets de poulet, sont moins riches en lipides (0,9\%) que les muscles rouges de la cuisse $(2,8 \%)$; la peau est nettement plus grasse : 26,9 \% (Ratnayake et al 1989, Leskanich et Noble 1997). Des valeurs similaires ont été observées plus récemment (Rabot et al 1999) sur des animaux d'âges et de souches différents.

Tableau 1. Teneurs en lipides totaux et en gras abdominal de la carcasse de différentes espèces aviaires (Leclercq 1989).

\begin{tabular}{|l|c|c|}
\hline $\begin{array}{l}\text { Espèce } \\
\text { (âge à l'abattage) }\end{array}$ & $\begin{array}{c}\text { Lipides totaux } \\
(\mathrm{g} / \mathrm{kg})\end{array}$ & $\begin{array}{c}\text { Gras abdominal } \\
(\mathrm{g} / \mathrm{kg})\end{array}$ \\
\hline $\begin{array}{l}\text { Poulet (45 jours) } \\
\text { - mâle }\end{array}$ & 155 & 27 \\
- femelle & 190 & 35 \\
\hline Dindonneau & & 10 \\
- mâle (112 jours) & 70 & 22 \\
- femelle (98 jours) & 155 & \\
\hline Canard & 180 & 35 \\
- mâle (84 jours) & 220 & 42 \\
- femelle (70 jours) & 220 \\
\hline
\end{tabular}

INRA Producti ons Ani males, décembre 2001

\section{2 / Alimentation et état d'engraissement}

En dehors des caractéristiques physiologiques propres à chaque espèce aviaire et qui régissent l'adiposité de la carcasse, l'état d'engraissement peut aussi être modulé par l'alimentation, en particulier par les concentrations énergétique et azotée de la ration et par leur rapport. II convient de garder à l'esprit que les modifications de la concentration énergétique de l'aliment sont, dans la majorité des essais publiés, réalisées par une substitution des glucides par des lipides alimentaires. Mais, puisque la valeur énergétique de l'aliment est toujours exprimée en énergie métabolisable (EM) et que les rendements de transformation de l'EM en EN (énergie nette) des lipides et des glucides ne sont pas les mêmes (Carré 2001), ces substitutions peuvent aboutir à des ratios EM/protéines identiques alors que les ratios EN/protéines ne le sont pas.

A cela s'ajoutent les modifications physiques de l'aliment (dureté, durabilité, pulvérulence, ... ) liées à la présence et/ou l'absence de certaines matières premières -dont les matières grasses- pouvant altérer la prise alimentaire et donc l'ingéré énergétique.

D'une façon générale, l'accroissement de la concentration énergétique de la ration, à rapport EM/protéines constant, a une incidence mineure sur la vitesse de croissance du poulet. Mais lorsque l'augmentation de la concentration énergétique de l'aliment s'obtient par un apport accru de lipides, le gain de poids et l'efficacité alimentaire sont améliorés. Les matières grasses auraient donc un effet spécifique sur la croissance, indépendant de l'effet de la concentration énergétique. Dans le même temps, I'ingéré énergétique augmente ainsi que les dépôts lipidiques (Fisher 1984, Leclerca 1986), mais la quantité de protéines corporelles ne change pas (J ackson et al 1982).

\section{3 / Synthèse des acides gras corporels}

Chez les oiseaux, la synthèse de novo des acides gras est très limitée dans le tissu adipeux et dans l'ovaire et c'est essentiellement le foie qui assure la synthèse endogène des acides gras à partir des glucides alimentaires. La dégradation de ces glucides aboutit à la formation d'acétylCoA puis à la synthèse de palmitate dans I'hépatocyte. Le palmitate est ensuite désaturé par une $\Delta 9$ désaturase et donne l'acide oléique. La synthèse d'acides gras saturés et monoinsaturés est principalement contrôlée par l'insuline et le glucagon. Les oiseaux, comme les mammifères, sont incapables de transformer ensuite l'acide oléique en $\alpha$-linoléique et $\alpha$-linolénique, en l'absence des désaturases correspondantes $\Delta 12$ et $\Delta 15$. Ils sont donc tributaires des apports alimentaires en ces acides gras. Ceux ci peuvent ensuite être convertis en acides à longue chaîne grâce à des désaturases ( $\Delta 5$ et $\Delta 6$ ) et élongases communes (figure 1).

La formation de ces acides gras est régulée par inhibition compétitive des enzymes et les 
Figure 1. Métabolisme des acides gras polyinsaturés (Calder 1998).

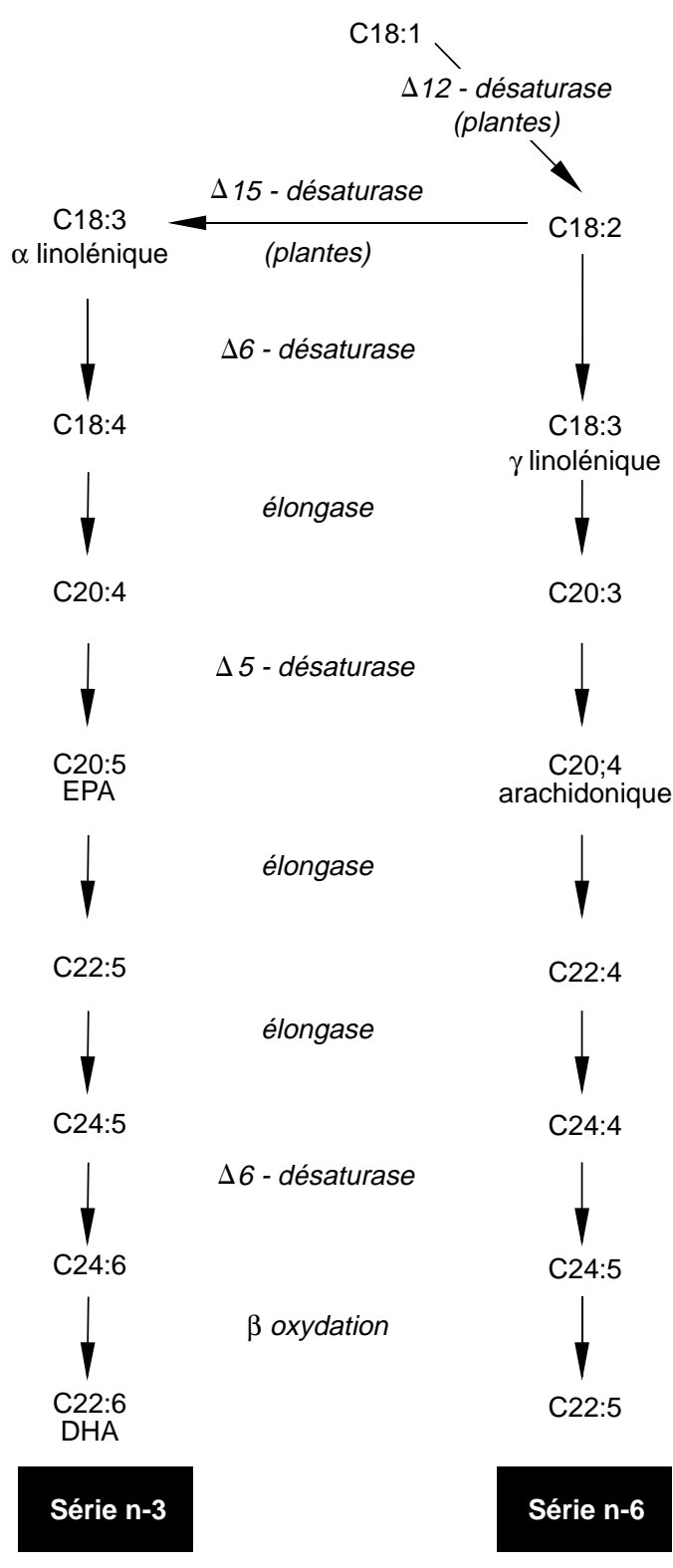

conversions au sein de chaque famille ( $n-3$ ou $n-6)$ dépendent des concentrations des substrats et des produits. Ainsi un excès d'acide linoléique limite la synthèse d'acide linolénique et inversement.

Ces acides gras polyinsaturés sont des inhibiteurs de la lipogenèse hépatique. D'une façon générale, l'ingestion d'un aliment riche en lipides inhibe la lipogenèse hépatique.

En définitive, la nature et les proportions relatives des acides gras déposés dans les tissus des oiseaux dépendent donc des apports en substrats alimentaires, glucides ou lipides, et de l'équilibre entre synthèse endogène hépatique et incorporation directe des acides gras alimentaires. Dans ces conditions, la composition en acides gras des tissus des oiseaux peut varier dans de larges proportions et être un bon indicateur de la nature des lipides ingérés.

\section{4 / Acides gras alimentaires et acides gras corporels}

\section{1 / Composition des lipides cor- porels en l'absence de lipides ajoutés à l'aliment}

Chez les poulets recevant depuis l'éclosion une alimentation sans addition de lipides, le taux de mortalité est élevé (plus de $50 \%$ ) et les tissus adipeux sont particulièrement peu développés. L'acide oléique est l'acide gras majoritaire de ces dépôts ( $58 \%$ ), suivi de l'acide palmitique $(25,1 \%)$, stéarique $(8,4 \%)$ et palmitoléique (6,4\%). La présence de C18:2 (linoléique) et C18:3 (linolénique) n'est pas détectée (Bottino et al 1970).

La distribution d'aliments non supplémentés en lipides mais constitués de céréales (maïs principalement) et de tourteaux induit des modifications profondes du profil en acides gras des tissus adipeux et de la carcasse. Ainsi apparaissent les acides linoléique ( $20 \%$ des acides gras totaux) et linolénique (moins de $1 \%$ ) principalement au détriment de l'acide oléique dont la proportion est ramenée à 39-42 \% (Marion et Woodroof 1963). Ces acides linoléique et linolénique proviennent des matières grasses contenues dans I'aliment et en particulier du maïs. De nombreux auteurs ont, par la suite, confirmé ces données, même si les profils en acides gras des carcasses et des dépôts lipidiques observés présentent de larges variations induites par la composition des aliments ingérés qui, outre les céréales et les tourteaux, pouvaient renfermer des farines animales ou de poisson (Edwards et al 1973, Blanch et al 1993, Scaife et al 1994, Leskanich et Noble 1997).

\section{2 / Modifications des lipides corporels par les lipides alimentaires}

Les modifications de profil en acides gras des lipides corporels sont encore plus évidentes lorsque les animaux recoivent des lipides alimentaires de composition particulière. Ainsi, les huiles de palme et de coprah accroissent les proportions des acides gras à chaîne courte et saturée; les graisses animales (suif et saindoux) enrichissent les dépôts lipidiques du poulet en C16:0 et C18:0. A l'inverse, avec les huiles végétales riches en acides gras polyinsaturés (colza, soja ou lin), ce sont les proportions d'acides polyinsaturés à 18 atomes de carbone qui augmentent. Les huiles marines, quant à elles, accroissent de façon très significative les proportions des acides à chaîne longue et polyinsaturée, C20:5, C22:5 et C22:6 de la série n-3 (Blanch é al 1993, Scaife et al 1994, Mossab et al 1999).

Le tableau 2 illustre les profils en acides gras de la cuisse de poulet selon qu'il ait ingéré différents aliments supplémentés ou non en lipides (Marion et Woodroof 1963). Dans cette étude ancienne, l'aliment de base renferme du maïs et du tourteau de soja, mais aussi de la farine de poisson, et les acides gras polyinsaturés ingérés sont incorporés dans 
Tableau 2. Influence de la nature des lipides ingérés sur la composition en acides gras (en \% des acides gras totaux) de la cuisse de poulet (Marion et Woodroof 1963).

\begin{tabular}{|l|c|c|c|c|c|c|c|c|c|}
\hline & C16:0 & C16:1 & C18:0 & C18:1 & C18:2 & C18:3 & C20:5 & C22:5 & C22:6 \\
\hline Aliment de base & 19,9 & 4,7 & 8,4 & 27,7 & 23,6 & 1,0 & 0,5 & 1,0 & 1,9 \\
+ huile de maïs (6 \%) & 16,2 & 2,4 & 6,0 & 24,7 & 38,7 & 1,8 & 0,8 & 0,7 & 0,7 \\
+ suif (6 \%) & 22,1 & 5,8 & 10,4 & 34,5 & 13,8 & 1,6 & 0,3 & 0,5 & 1,4 \\
+ huile de maïs (5 \%) & & & & & & & & & \\
+ huile de poisson (1\%) & 17,4 & 4,4 & 5,2 & 28,7 & 33,8 & 1,0 & 1,0 & 1,3 & 1,6 \\
+ suif (5\%) & & & & & & & & & \\
+ huile de poisson (1\%) & 22,6 & 5,3 & 10,6 & 29,8 & 15,3 & 1,9 & 1,7 & 2,5 & 2,4 \\
\hline
\end{tabular}

les tissus musculaires. L'ajout d'huile de maïs à l'aliment de base augmente la quantité de C18:2 incorporée. Avec un apport de suif, le muscle est plus riche en C16:0, C18:0 et C18:1 et plus pauvre en C18:2. La substitution d'une partie de l'huile de maïs ou du suif par de I'huile de poisson enrichit le tissu musculaire en acides gras polyinsaturés à chaîne longue. Cette propension des volailles à incorporer les acides gras alimentaires dans leurs tissus a été utilisée pour apporter au consommateur les acides gras préconisés par le corps médical, en particulier ceux de la série n-3 (voir revues de Hargis et Van Elswyk 1993 et de Leskanich et Noble 1997).

Beaucoup plus récemment, afin de quantifier l'incidence de la nature des lipides alimentaires sur la composition et la qualité de la viande de poulet, un programme financé par l'Acta, les ministères de l'Agriculture et de la Pêche et de l'E nseignement Supérieur et de la Recherche a été développé entre différents partenaires (CCPA, Cemagref, Cetiom, Evialis, Glon-Sanders, Inra, Iterg, Onidol, Ucaab, Primex-Unicopa). Cing essais successifs ont été réalisés mettant en œuvre plusieurs milliers de poulets. Tous recevaient pendant la période de démarrage ( $0-21$ jours) un aliment commun renfermant $5 \%$ d'huile de maïs. Les poulets étaient ensuite séparés en différents lots et alimentés avec un aliment contenant $8 \%$ d'un mélange de différentes matières grasses: suif, huiles de palme, de coprah, de tournesol, de tournesol oléique, de maïs et de lin. Ces corps gras étaient associés afin de faire varier les teneurs en C18:2 alimentaire de 20 à $60 \%$ à C18:3 constant ( $2 \%$ ) et celles du C18:3 de 2 à $10 \%$ à C $18: 2$ constant (40\%). Le suif servait de matière grasse saturée de référence, puis il a été remplacé par des huiles végétales du fait de son interdiction en alimentation animale. Au total, 35 aliments ont été utilisés.

Pour chaque essai, les performances zootechniques des animaux ont été mesurées et, après abattage, la qualité de présentation des carcasses a été appréciée par une notation allant de 1 (graisse molle et huileuse) à 5 (graisse ferme et sèche). Les profils en acides gras ont été déterminés sur un mélange de gras abdominaux prélevés sur des poulets issus du même lot ainsi que sur les muscles de la cuisse et du filet. La cinétique d'incorporation des acides gras dans les différents tissus a fait l'objet de mesures spécifiques.

D'une façon générale, les performances de croissance ont été très peu modifiées par la INRA Producti ons Ani males, décembre 2001 nature des lipides ingérés. La cinétique d'incorporation des acides gras dans les triglycérides du Pectoralis major est illustrée par la figure 2. Le profil en acides gras est profondément modifié par la nature et la quantité relative des différents acides gras ingerés, mais n'évolue pratiquement plus après deux semaines de distribution des aliments expérimentaux.

La composition en acides gras du gras abdominal est également étroitement liée à celle des lipides alimentaires (figure 3). Les coefficients de corrélation calculés pour chaque acide gras sont élevés et varient de 0,92 pour les acides stéarique et oléique à 0,98 pour l'acide linoléique. II en est de même des acides gras incorporés dans les triglycérides et les phospholipides du muscle et il est ainsi possible de prédire (Gandemer et al 1999, Viau et al 1999) les quantités déposées (Y) en fonction des quantités $(X)$ ingérées.Pour la quantité de $C 18: 2$ déposée dans la cuisse :

$Y=0,429 X+12,36 \quad\left(R^{2}=0,999\right)$

Figure 2. Cinétique d'incorporation des C18:2 et C18:3 dans les triglycérides du Pectoralis major de poulet en fonction des apports alimentaires en ces acides gras et de l'âge (Viau et al 1999, Gandemer et al 1999).

C18:2
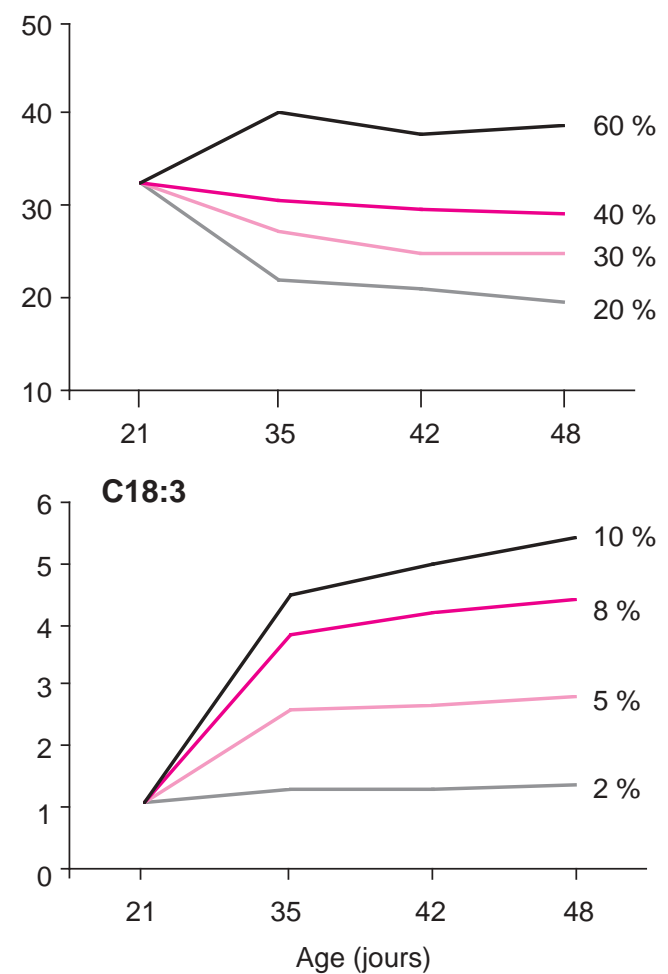
Figure 3. Relations entre les acides gras alimentaires $(x)$ et ceux du gras abdominal du poulet $(y)$.
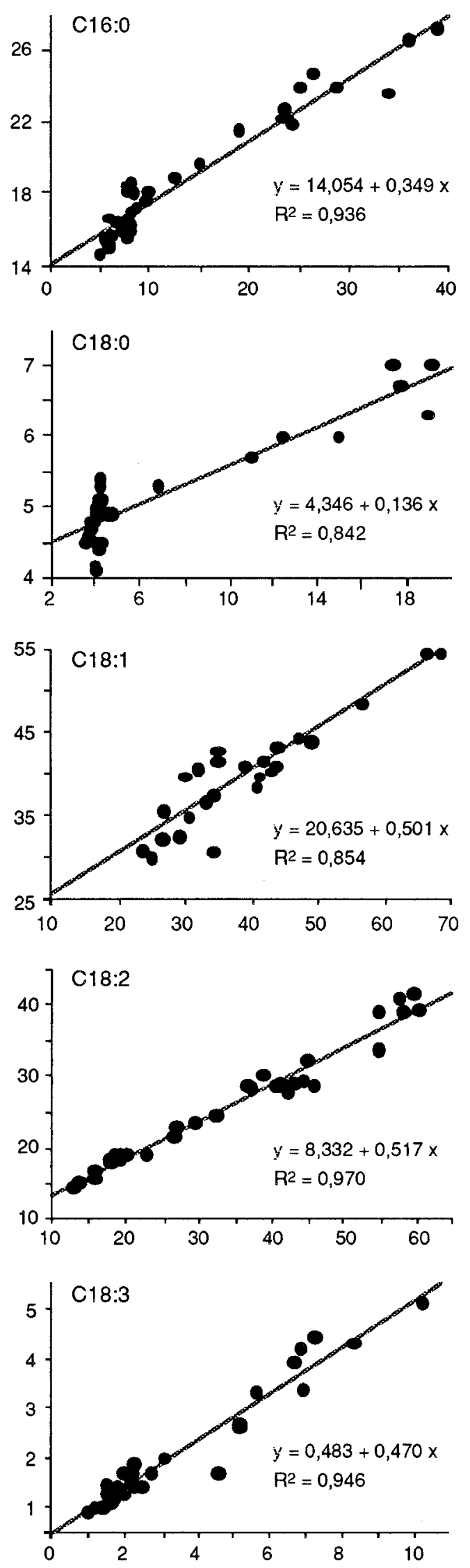

La qualité visuelle de la carcasse est corrélée positivement aux teneurs en acides gras des dépôts lipidiques et donc à celles de l'aliment : $r=0,59$ et 0,58 pour $C 16: 0$ et $\mathrm{C} 18: 0$ respectivement. Elle est dégradée par les acides gras polyinsaturés alimentaires : linoléique $(r=-0,61)$ et linolénique $(r=-0,42)$. Le rôle de l'acide oléique est négligeable $(r=-0,16)$. Ces relations permettent d'établir une prédiction de la note de la carcasse:

Note de carcasse $=5,76-0,11[\mathrm{C} 18: 3]-0,04$ [C18:2] - 0,03 [C18:1]

$r=0,8$

Cette équation permet de calculer les teneurs en acides gras alimentaires à ne pas dépasser afin de ne pas dégrader la qualité de la carcasse. A terme il est possible d'envisager d'utiliser de telles équations dans les programmes de formulation des aliments composés pour les volailles.

\section{Conclusion}

Les viandes de volailles sont appréciées des consommateurs et du corps médical car elles ont la réputation d'être pauvres en lipides et d'apporter des acides gras insaturés favorables à la santé. Ces viandes sont réellement pauvres en lipides si I'on considère les muscles principalement consommés (filets et cuisses). II n'en est pas de même si l'on prend en considération l'ensemble de la carcasse qui peut alors renfermer des quantités importantes de gras, or ce gras est une perte pour l'abattoir et le consommateur.

Des conduites alimentaires spécifiques, associées à l'utilisation de génotypes aviaires sélectionnés contre l'engraissement, peuvent facilement moduler l'état d'adiposité des oiseaux. Ainsi, l'accroissement des apports protéiques ou la réduction de l'ingéré énergétique vont limiter l'adiposité des carcasses des volailles. A l'inverse, il ne semble pas que l'incorporation pratiquement systématique de matières grasses dans I'aliment soit directement responsable d'une adiposité accrue des carcasses. Mais leur emploi a sans aucun doute contribué à accroître la densité énergétique des rations, donc l'ingéré énergétique et donc l'engraissement.

Comme les mammifères, les oiseaux sont incapables de synthétiser les acides gras polyinsaturés qu'ils doivent trouver dans la fraction lipidique de leur alimentation, qu'il s'agisse de corps gras ou de graines oléagineuses. Ces acides gras inhibent la lipogenèse hépatique et se substituent en partie à l'acide oléique dans les tissus adipeux de réserve et dans les phospholipides du muscle. II est ainsi facilement possible d'enrichir les viandes de volailles en certains acides gras et en particulier en oméga 3 grâce à l'incorporation dans l'aliment d'huiles de poisson, de colza ou de lin. Cependant cet enrichissement se traduit par l'apparition de gras corporels plus fluides, préjudiciables à la qualité visuelle et technologique des produits animaux et qu'il convient de limiter par un apport accru de lipides saturés. La qualité organoleptique de carcasses ainsi enrichies peut aussi être altérée puisque ces acides gras très insaturés sont facilement 
oxydables et il convient de les protéger par des apports accrus d'antioxydants tels la vitamine $\mathrm{E}$.

En définitive, loin de dégrader la qualité des viandes de volailles, les lipides alimentaires peuvent au contraire contribuer au dévelop- pement des productions aviaires en véhiculant jusqu'au consommateur les acides gras préconisés par le corps médical, sous réserve de ne pas transformer le poulet ou la dinde en un médicament dont la qualité organoleptique serait devenue déplorable.

\section{Références}

Alleman F., Bordas A., Caffin J.P., Daval S., Diot C., Douaire M., Fraslin J.M., Lagarrigue S., Leclercq B., Tixier-Boichard M., 1999. L'engraissement chez le poulet aspects métaboliques et génétiques. INRA Productions Animales, 12, 257-264

Berri C..J ehl N., 2001. Facteurs de variation de la qualité technologique et organoleptique des viandes de poulets. In : 4èmes J ournées de la Recherche Avicole, Nantes (FRA), 2001/03/27-29, 245-252. ITAVI, Paris

Blanch A., Barroeta A.C., Puchal F., 1993. Effects of dietary linseed oil and palm oil of fatty acid profile of carcass fat depots in broiler chickens. In : Quality of poultry meat, Congrès WPSA, Tours (France), 1993/10/04-08, 86-92.

Bottino N.R., Anderson R.E., Reiser R., 1970. Animal endogenous triglycerides: 2 . Rat and chicken adipose tissue. Lipids, 5, 165-170.

Calder P.D., 1998. Dietary fatty acids and the immune system. Nutr. Rev., 56, 570-583.

Carré B., 2001. Evaluation de la valeur énergétique des aliments des oiseaux d'élevage. In : 4èmes | ournées de la Recherche Avicole, Nantes (FRA), 2001/03/27-29, 123-130. ITAVI, Paris

Edwards H.M.Jr, Denman F., Abou-Ashour A., Nugara D., 1973. Carcass composition studies. 1. Influence of age, sex and type of dietary fat supplementation on total carcass and fatty composition. Poultry Sci., 52, 934-948.

Fischer C., 1984. Fat deposition in broiler. In : J. Wiseman (ed), Fats in animal nutrition, 437-470. Butterworth, Nottingham (UK).

Gandemer G., Viau M., Maillard N., Lessire M., J uin H., 1999. Lipides alimentaires et qualité de la viande de poulet : influence de l'apport de quantité croissante d'acide linolénique (18:3 n-3). In : 3èmes Journées de la Recherche Avicole, Saint-Malo (Fra) , 1999/03/23-25, 403406. ITAVI, Paris.

Hargis P.S., Van Elswyk M.E., 1993. Fatty acid modification of poultry products. World's Poult. Sci., 49, 251-264.

Jackson S., Summers J.D., Leeson S., 1982. Effect of protein and energy on broiler carcass composition and efficiency of nutrient utilization. Poultry Sci., 61, 2224-2231.
Larbier M Leclercq B , 1992. Nutrition et alimentation des volailles. INRA Editions, Paris, 352p.

Leclercq B., 1986. Energy requirements of avian species. In : C. Fisher and K.N. Boorman (eds), Nutrients requirements of poultry and nutritional research, 125141. Butterworths, London (UK).

Leclercq B., 1989. Possibilités d'obtention et intérêt des génotypes maigres en aviculture. INRA Productions Animales, 2, 275-286.

Leskanich C.O., Noble R.C., 1997. Manipulation of the n-3 polyunsaturated fatty acid composition of avian egg and meat. World's Poultry Sci., 53, 155-184.

Lessire M., 1995. Qualité des viandes de volaille : le rôle des matières grasses alimentaires. INRA Productions Animales, 8, 335-340.

Marion J.E., Woodroof J .G., 1963. The fatty acid composition of breast, thigh and skin tissues of chicken broilers as influenced by dietary fats. Poultry Sci., 42, 1202-1207.

Mossab A., Lessire M., Hallouis J .M., Hermier D., 1999 Using dietary fats to improve polyunsaturated fatty acid content of poultry meat. In : L.G. Cavalchini and D. Baroli (eds), 14th European Symposium on the Quality of Poultry Meat, Bologna (ITA), 1999/09/19-23, 159-166. WPSA, Italian Branch, Bologna (ITA).

Rabot C., Gandemer G., J uin H., Meynier A . Lessire M. 1999. Importance relative de la souche, de l'aliment et de l'âge sur les caractéristiques lipidiques et sensorielles des muscles chez le poulet. In: 3èmes Journées de la Recherche Avicole, Saint-Malo (FRA), 1999/03/23-25, 447450. ITAVI, Paris.

Ratnayake W.M.N., Ackman R.G., Hulan H.W., 1989 Effect of redfish meal enriched diets on taste and n-3 PUFA of 42 days old broiler chickens. J. Sci. Food Agric., 49, 59-74.

Scaiffe J.R., Moyo J ., Galbraith H., Michie W., Campbell V., 1994. Effect of different dietary supplemental fats and oil on the tissue fatty acid composition and growth of female broilers. Br. Poultry Sci., 35, 107-118.

Viau M., Gandemer G., Meunier F., Lessire M., J uin H., 1999 Lipides alimentaires et qualité de la viande de poulet: influence de l'apport de quantité croissante d'acide linoléique $(18: 2 n-6)$. In : $3^{\text {emes }}$ J ournées de la Recherche Avicole, SaintMalo (FRA), 1999/03/23-25, 259-362. ITAVI, Paris.

\section{Abstract}

Dietary fats and poultry fatty acid composition.

Poultry meat has gained consumer approval and is recommended by dieticians because it is lean, yet contains a high proportion of unsaturated fatty acids. However, the leanness of poultry meat varies with species, age and sex, while the amount of fat depends on a number of dietary criteria. Fat is principally added to feed to increase its energy content and to improve production performances. It has little effect on the total fat carcass content when the diet is balanced, and in parINRA Producti ons Ani males, décembre 2001 ticular when the protein to energy ratio is held constant. On the contrary, the type of fat added has a profound influence on carcass fatty acid composition. Thus, it is possible to modify the carcass fatty acid spectrum in chickens in response to the quality requirements of slaughterhouses or consumers. This article summarizes the possible variations in poultry tissue fatty acids in relation to the profile of ingested lipids.

LESSIRE M., 2001. Matières grasses alimentaires et composition lipidique des volailles. INRA Prod. Anim., 14, 365-370. 